\title{
La santé maternelle des « Africaines » en Île-de- France : racisation des patientes et trajectoires de soins
}

Reproductive Health of "African"-perceived Women in Paris Area: Racial Categorisations and Medical Course

La salud materna de las "africanas» en Île-de-France: racialización de las mujeres y trayectorias de cuidados

\section{Priscille Sauvegrain}

\section{(2) OpenEdition}

\section{Journals}

Édition électronique

URL : https://journals.openedition.org/remi/5902

DOI : 10.4000/remi.5902

ISSN : $1777-5418$

\section{Éditeur}

Université de Poitiers

Édition imprimée

Date de publication : 1 juin 2012

Pagination : 81-100

ISBN : 979-10-90426-04-7

ISSN : 0765-0752

\section{Référence électronique}

Priscille Sauvegrain, «La santé maternelle des « Africaines » en Île-de-France : racisation des patientes et trajectoires de soins », Revue européenne des migrations internationales [En ligne], vol. 28 - n² | 2012, mis en ligne le 10 octobre 2012, consulté le 14 avril 2022. URL : http://journals.openedition.org/remi/ 5902 ; DOI : https://doi.org/10.4000/remi.5902 


\title{
La santé maternelle des «Africaines » en Île-de-France : racisation des patientes et trajectoires de soins
}

\author{
Priscille SAUVEGRAIN ${ }^{1}$
}

\begin{abstract}
J'ai beaucoup de copines, de belles-sœurs, etc. qui disent qu'on oblige
《les femmes africaines à faire des césariennes pour limiter les naissances ». Fatoumata M., dont nous avons recueilli le récit de vie, a ensuite longuement développé ce thème. Sur les divers forums de discussion consultés sur Internet, le ton adopté est encore plus direct : "Je voulais dénoncer cette pratique fasciste qui consiste à soumettre systématiquement les femmes africaines à la césarienne au moment de l'accouchement. Sur dix femmes africaines qui accouchent, six le font par césarienne. Il paraît que c'est pour limiter la fécondité des femmes noires... $»^{2}$. Ce type de propos ou de questions sur les taux de césarienne revient de façon récurrente depuis le début des années 1980 (Poiret, 1996 ; Quiminal, communication personnelle) et met en cause les pratiques cliniques françaises à l'égard des femmes d'origine africaine dans les services de gynécologie-obstétrique. Au sein de ces derniers, ni des taux différents de césariennes ni cette perception qu'ont les femmes «Africaines $»^{3}$ d'une discrimination ne sont connus, leur mauvaise acceptation des césariennes étant généralement qualifiée de « culturelle » (Mabe, 1985 ; Ba, 2000 ; Moro, 2002). Et, il est utile de le préciser, personne ne souhaite contrôler leur fertilité.
\end{abstract}

1 Docteure en sociologie (URMIS) et sage-femme (APHP Pitié-Salpêtrière) ; priscille.sauvegrain@ gmail.com

2 Propos extrait le 1er février 2012 d'un forum de discussion sur le site Grioo.com (http://www. grioo.com/forum/viewtopic).

3 Dans le corps du texte et pour bien signifier qu'il s'agit d'une catégorie construite, nous encadrerons le terme «Africaines » de guillemets. En revanche et pour ne pas alourdir les propos, nous ne préciserons pas toujours « catégorisées comme». 
Nous nous proposons néanmoins, dans cet article, d'examiner les pratiques médicales sous l'angle de la construction par les soignants de catégories racisantes ${ }^{4}$ de patientes.

Peu d'études cliniques françaises portent sur la santé périnatale des femmes en fonction de leurs origines réelles ou supposées. Dans plusieurs pays, ces catégories sont aisément utilisées, tant d'ailleurs par la médecine (Carde, 2011) que par d'autres disciplines, et font l'objet d'enregistrements institutionnels dans les instruments de comptage de la population (Schor, 2003 ; Simon, 1997). En France, en dépit des tentatives de la pharmacologie, des biotechnologies et de la génétique (Aboukra et Noiville, 2011 ; Henn, 2011 ; Roberts, 2011), la tradition politico-juridique de ne pas prendre en compte les origines, au nom de l'indivisibilité de la nation, interdit leur institutionnalisation.

Au début des années 1980, des équipes médicales et des responsables politiques français ont été interpellés par des leaders associatifs (Poiret, 1996), mais aussi par les autorités consulaires maliennes (Sauvegrain, 2010) qui dénonçaient les taux particulièrement élevés de césariennes de leurs ressortissantes accouchant en France. Le 7 juin 1984, Georgina Dufoix, alors ministre de la Santé, a organisé avec l'appui du Comité médicosocial pour la santé des migrants, une réunion avec des équipes médicales en vue d'établir un « bilan des problèmes posés par et aux femmes africaines noires à l'occasion de leur grossesse et proposer des solutions à court et moyen termes $»^{5}$.

Les études cliniques menées à l'époque ont confirmé une surreprésentation des femmes migrantes originaires d'Afrique de l'Ouest parmi les femmes ayant accouché par césarienne. En 1979, une première équipe hospitalière parisienne (Pigné et al., 1984 et 1985) a trouvé un taux de césariennes, pour les femmes originaires du Mali et du Sénégal, deux fois supérieur au taux moyen de césariennes en France (23\% contre $13 \%$ en moyenne). Puis elle a montré qu'en adoptant une attitude obstétricale plus attentiste, il était possible d'abaisser ce taux élevé. Si quelques travaux indiquent une baisse dans les années qui suivirent la réunion du 7 juin 1984 et la publication de l'étude de Pigné (Col, 1989 ; Pambou et al., 1996), les taux ne tardèrent pas à remonter au tournant des années 1990. Une étude toulousaine rapporte, entre 1988 et 1994, des taux de césarienne très significativement supérieurs pour les femmes originaires d'Afrique noire, avec $34 \%$ versus $19 \%$ pour la population générale (Gayral-Taminh et al., 1999). Une autre étude menée au CHU de Rouen sur la période 1998-2000 montre que le taux de césarienne pour les femmes d'origine africaine est de $38 \%$ quand il est de 19,7\% pour les autres femmes (Benoit, 2004). Si les études des années 1970-1980 portaient plutôt sur des

4 Par ce terme, nous entendons ancrer clairement les catégories liées à l'origine - réelle ou supposée - des minoritaires dans des relations sociales. Il s'agit bien de processus de désignation et/ou d'affirmation identitaires qui n'ont de sens que par, et dans, les relations et rapports sociaux dans lesquels ils se construisent. L'utilisation des suffixes « -ant» (racisant) ou « ion » (racisation) exprime la dimension dynamique, et non statique, de ces phénomènes. Nous ne parlerons pas dans cet article d'ethnicisation, qui renvoie plutôt à des représentations culturalisantes, non pas que les femmes catégorisées comme «Africaines » ne soient jamais désignées comme ayant des pratiques culturelles spécifiques, elles le sont pour tout ce qui a trait au rôle maternel. Mais, en ce qui concerne les soins prénataux et l'accouchement, c'est davantage en termes de différences biologiques et physiologiques que la différence est saillante dans les propos des soignants.

5 Les interventions de cette journée ont été intégralement publiées l'année suivante dans le numéro 44 de Migrations Santé, $3^{\text {ème }}$ trimestre 1985. 
femmes immigrées classées par pays de l'Afrique subsaharienne, les études cliniques plus récentes traitent souvent de «femmes d'origine africaine » ou de « Noires Africaines » sans préciser comment ces catégories sont construites ni qui elles englobent.

Nous avons tenté, dans le cadre de notre recherche doctorale (Sauvegrain, 2010), de comprendre la variation des taux de césarienne des «Africaines » au cours des trois dernières décennies. Elle semble associée à des observations cliniques qui les distinguent de façon récurrente des autres femmes. Au cours de la décennie 1970, les «Africaines » ont été très fortement césarisées ${ }^{6}$ en raison d'une " mécanique obstétricale " ${ }^{7}$ différente. Ainsi, on trouve des descriptions cliniques qui les doteraient de bassins plus petits que ceux des autres femmes. Le taux de césarienne, à cette période, est justifié - ce qui ne veut pas dire que cette justification clinique ait disparu - par le « caractère du bassin qui présente une forme androïde » (Pambou et al., 1996 : 375). Dans les années 1990, c'est la prévention de la transmission mère-enfant du VIH/Sida qui va venir justifier l'établissement d'un protocole de césarienne systématique pour toutes les femmes séropositives. L'arrivée des antirétroviraux en 1997 et l'observation de « séroconversion » chez les nourrissons dans les premiers mois de la vie feront reculer la systématisation de ce protocole, au profit d'une approche plus individualisée (Delfraissy, 1999) ${ }^{8}$.

Mais un autre protocole vient, dans les années 2000, signer la racisation des femmes «Africaines » et, par extension, des femmes « Noires »". Une caractéristique clinique concernant leur temps de gestation les singulariserait. Cette donnée « probante $»^{10}$ s'appuie sur une étude de cohorte qui rapporte que les patientes " d'origine africaine », ou « Blacks » ${ }^{11}$ aux États-Unis, ont une durée de grossesse plus courte de cinq jours que toutes les autres femmes (Papiernick et al., 1990). Cette étude, citée comme une référence en France où aucune autre n'a été menée sur le sujet, affirme que cette différence de temps de gestation serait surtout génétique, et parfois liée aux conditions socio-économiques de

6 Terme consacré en clinique, que nous reprenons pour faciliter la lecture.

7 Ce terme, également consacré en clinique, désigne les différentes étapes d'engagement et de progression du fotus dans le bassin maternel.

8 L'évolution de la voie d'accouchement des femmes séropositives pour le VIH-Sida dans le but de réduire la transmission materno-fœtale est un sujet en soi. Elle ne sera donc pas traitée dans cet article.

9 Ce terme est également une construction sociale. S'il y a un relatif consensus, dans le monde scientifique, pour dire que les races n'existent pas, cela n'empêche pas les catégories racisantes de rester performatives (Guillaumin, 1972).

10 Depuis les années 1980, les protocoles adoptés dans les services de santé sont adossés à ce qu'il est convenu d'appeler en France « les données probantes », traduction de la notion anglaise «Evidence Based Medicine » (EMB), largement diffusées dans le monde médical au travers de banques de données bibliographiques, notamment de la Librairie Cochrane qui intègre plusieurs bases de données : CDSR (Cochrane Database of Systematic Review) ; DARE (Database of Abstracts of Reviews of Effectiveness) est produite par le National Health Service Centre for Reviews and Dissemination (CDR) de l'Université de York (UK) ; CCTR (Cochrane Controlled Trials Register) ; CRMD (Cochrane Review Methodology Database) ; NHS EED (NHS Economic Evaluation Database); HTA (Health Technology Assessment Database).

11 Une partie de l'étude porte sur une cohorte américaine, ce qui explique l'usage de cette catégorie, dont il faut ici rappeler qu'elle est institutionnalisée et complètement admise en épidémiologie aux États-Unis. 
vie, sans plus de détails ${ }^{12}$. Ses auteurs proposent, en conclusion, d'anticiper d'une semaine la prise en charge de la fin de grossesse de ces patientes. Ceci n'a pas été immédiatement suivi d'effets, mais, dans les années 2000, de très nombreuses maternités françaises ont mis en place un « protocole de terme ethnique »-parfois appelé " protocole de terme couleur »- qui consiste en une surveillance spécifique de la fin de grossesse des femmes repérées comme « Noires ». Aucune société savante n'avait émis de recommandations dans ce sens. D'ailleurs, en décembre 2011, le Collège national des gynécologues et obstétriciens français a précisé que ces données n'avaient pas été validées (CNGOF, 2011).

Le traitement particulier réservé aux parturientes « Africaines » ou « Noires », via ces protocoles cliniques, peut avoir un impact sur des taux de césarienne plus élevés pour elles que pour les femmes d'origine française, ou d'autres origines étrangères. Il mérite donc de plus amples investigations. En effet, la connaissance des faits et processus qui sont à l'origine de l'état de santé et des trajectoires de soins de ces femmes nous semble essentielle, car celles qui sont originaires d'Afrique Subsaharienne et qui ont une nationalité étrangère ont, en France et à l'heure actuelle, cinq fois plus de risque de mourir en couches que les autres femmes ${ }^{13}$, et leurs décès sont associés à une qualité de soins dite « non optimale » dans $78 \%$ des cas (Bouvier-Colle et al., 2008).

En nous appuyant sur les résultats d'une étude menée dans des services de gynécologie-obstétrique franciliens entre 2006 et 2010 (Sauvegrain, 2010), nous montrerons combien la racisation des patientes catégorisées comme "Africaines » ou " Noires », au nom de caractéristiques biologiques et génétiques qui les singulariseraient, est efficiente. Ce processus est d'ailleurs observé dans d'autres secteurs de la société française (Cognet et Bascougnano, à paraître ; Poiret, 1996, 2010 et 2011). Dans un premier temps nous rappellerons notre méthodologie, puis nous présenterons les résultats des analyses statistiques effectuées à partir des données colligées dans les dossiers des femmes ayant accouché dans un CHU parisien en 2006. Après avoir comparé les taux de césarienne des femmes de la cohorte selon leurs origines réelles ou supposées, nous examinerons ce qui, en l'état des données disponibles, peut expliquer les écarts de taux de césariennes entre les femmes susceptibles d'être catégorisées comme «Africaines » et les autres. Dans une troisième partie, nous interrogerons les processus de catégorisation de ces femmes, en nous appuyant sur les entretiens menés avec des cliniciens et sur une analyse bibliographique. Nous verrons comment les représentations de leur altérité alimentent la catégorie " Africaines » et la rendent opératoire pour orienter et justifier les décisions et les pratiques cliniques, en salle d'accouchement ou dans le cadre du " protocole de terme ethnique ". Ce dernier nous amènera à questionner une éventuelle discrimination de ces femmes dans les soins.

12 D'autres articles sur le même sujet sont beaucoup plus prudents dans leurs conclusions et soulignent surtout le poids des variables socio-économiques dans une éventuelle variation du terme de grossesse (Alexander et al., 1993 ; Patel et al., 2003).

13 Deux principales causes de décès sont mises en évidence : les femmes d'Afrique subsaharienne présentent un risque sept fois supérieur à celui des Françaises de mourir des complications d'une hypertension liée à la grossesse (première cause de décès dans cette population) et un risque neuf fois supérieur pour les infections (cause rare de décès). 


\section{L'ENQUÊTE MENÉE DANS DES SERVICES DE GYNÉCOLOGIE- OBSTÉTRIQUE FRANCILIENS}

Notre étude combine approches quantitative et qualitative. L'étude quantitative a été menée à partir de 2111 dossiers ${ }^{14}$ de femmes ayant accouché en 2006 dans la maternité d'un CHU parisien. Ce choix est justifié par le poids des femmes immigrées dans la population régionale : la moitié des femmes ayant accouché dans cet hôpital en 2006, étaient des immigrées et, parmi elles, 40,1\% venaient d'Afrique subsaharienne ${ }^{15}$ alors que l'enquête française de périnatalité de 2010 relève que sur l'ensemble des femmes accouchant en France chaque année, 13,3\% sont immigrées, et 2,8 \% originaires d'Afrique subsaharienne (Blondel et al., 2011). Il s'agit d'une maternité qui dispose d'une unité de néonatologie. Ses usagères habitent le quartier ou sont adressées de plus loin pour un suivi médical particulier, ce double recrutement entraînant une certaine mixité sociale.

Les données disponibles ont été classées pour permettre des analyses statistiques multivariées ${ }^{16}$. Les variables dépendantes concernent les trajectoires de soins : le déclenchement ou non de l'accouchement, son terme et son mode (voie basse ou césarienne), et l'état de santé des nouveau-nés à la naissance. Pour vérifier nos différentes hypothèses, et surtout d'éventuelles variations en matière de voie d'accouchement, nous souhaitions tout d'abord disposer de groupes de patientes établis selon leur état de santé. Les variables explicatives ont été rassemblées sous forme de " profils médicaux » pour la grossesse (différenciant les grossesses normales des grossesses pathologiques ${ }^{17}$, sur le versant maternel et/ou fotal), et pour le travail ${ }^{18}$, au cours duquel de nouvelles situations pathologiques peuvent survenir, pour la mère ou pour le fœtus. Ensuite, nous voulions tester quantitativement le rôle que pouvaient jouer les catégorisations racisantes. Dans la mesure où il appartient aux soignants de repérer les patientes pour lesquelles des protocoles spécifiques sont préconisés (en particulier celui de « terme ethnique »), nous disposions d'une variable rendant compte d'une assignation à une catégorie racisante. Les origines supposées des

142248 femmes ont accouché dans ce service cette année-là, mais 137 dossiers n'étaient pas exploitables. Les données anonymisées ont été enregistrées et traitées sur le logiciel SPSS.

15 Les pays de naissance de ces femmes sont l'Angola, le Bénin, le Burkina Faso, la Côte d'Ivoire, le Cap-Vert, le Cameroun, la Centrafrique, la République démocratique du Congo, le Gabon, la Gambie, la Guinée, la Mauritanie, le Mozambique, le Nigeria, le Rwanda, le Tchad et le Togo. Leur date d'arrivée en France n'est que rarement renseignée.

16 Les éléments renseignés dans la base de données ne nous permettaient pas d'établir les profils socio-économiques des patientes, ce que nous déplorons.

17 Cinq pathologies maximum par patiente étaient disponibles dans la base de données. Elles peuvent être antérieures à la grossesse ou apparaître au cours de celle-ci et sont de gravités diverses. Mais toutes ont une influence sur la trajectoire de soins en maternité et peuvent avoir une répercussion sur le bien-être fotal. Seules les patientes présentant une pathologie de l'hémoglobine isolée n'ont pas été incluses dans la catégorie des femmes porteuses de pathologies. En effet, nous pensions, en nous appuyant sur la prévalence régionale de la pathologie, que la donnée était mal renseignée, amalgamant des femmes porteuses et des femmes malades.

18 Le travail désigne la phase de contractions utérines et de dilatation cervicale préalable à tout accouchement par voie vaginale. 
femmes ${ }^{19}$, mais aussi celles de leurs parents, sont inscrites sur les registres d'accouchement, ou sur les dossiers obstétricaux dans la case « origine », ou sous forme de corrections faites à la rubrique «nationalité ». Nous avons ainsi pu identifier l'émergence d'une catégorie de femmes classées comme «Africaines ». Enfin, nous avons voulu savoir si le fait d'être née en France avait une influence en matière de santé périnatale. À partir des lieux de naissance, nous avons créé une variable rendant compte de la migration, en deux

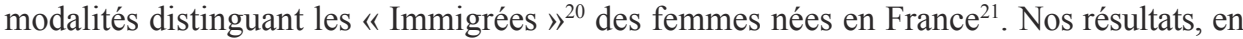
dépit de leur caractère parfois exploratoire, satisfont aux principaux critères de validation statistique, comme en témoignent les différents tests d'association qui leur ont été appliqués ${ }^{22}$.

Les données quantitatives ont été enrichies par une enquête qualitative qui combine des phases d'observation participante dans l'hôpital dans lequel nous exerçons comme sage-femme, et cinquante-six entretiens semi-directifs dits de conversation (Olivier de Sardan, 1995) avec des soignants de diverses professions exerçant dans différentes maternités franciliennes.

\section{LES RÉSULTATS STATISTIQUES}

En 2006, dans la maternité étudiée, 21,6\% des accouchements se sont déroulés par césarienne ${ }^{23}$, taux qui rejoint la moyenne nationale de $21 \%$ selon l'enquête de périnatalité de 2010 (Blondel et al., 2011). Mais le taux de césarienne des seules «Africaines » est de $31,3 \%$ alors que pour les autres femmes, quelles que soient leurs origines, il n'est que de 20,2 \%. Autrement dit, avant contrôle de toute autre variable, ces femmes sont significativement plus souvent césarisées que les autres.

La prise en compte de leur lieu de naissance permet d'observer que ce sont surtout les femmes nées dans un pays d'Afrique subsaharienne et ayant migré en France, quels que soient leurs nationalités et leurs statuts juridiques sur le territoire métropolitain, qui ont un taux de césarienne nettement plus élevé : 33,3\% contre 19,3\% pour les femmes nées en France, toutes origines confondues et 20,9\% pour les femmes nées à l'étranger

19 La question de leurs origines géographiques et de celles de leurs parents est posée aux femmes lors de leur première consultation et nous avons observé que cette donnée était méticuleusement renseignée dans cette maternité.

20 Il s'agit ici de la catégorie démographique introduite par l'INSEE en 1990 : nées étrangères à l'étranger.

21 Notre étude comprenait d'autres sous-groupes, par exemple des femmes immigrées d'autres pays d'Europe, ou d'autres continents, mais nous n'avons que rarement trouvé des différences significatives entre elles et le groupe des « Françaises nées en France de parents nés en France ». Il n'est donc pas pertinent d'éclater ces catégories dans la présentation des résultats.

22 Les résultats statistiques d'association sont rendus à l'aide d'odds ratios avec un intervalle de confiance de $95 \%$ et le test du $\mathrm{Khi}^{2}$ correspondant. Pour mesurer la force de la dépendance, les tests de recherche d'association suivants ont également été éprouvés : coefficient de contingence, Phi et V de Cramer, Lambda pour les variables nominales (dont les différentes données ne sont pas liées) ; taux de corrélation et taux de Kendall pour les ordinales (dont les différentes données peuvent être ordonnées). Les tests de probabilité établis grâce au $\mathrm{Khi}^{2}$ sont considérés comme significatifs pour $\mathrm{p}$ inférieur à 0,05 ce qui est une limite admise pour les sciences sociales et les données qualitatives (Trudel et Antonius, 1999). Puis les résultats significatifs ont été soumis aux tests de régression logistique pour vérifier les corrélations variable par variable.

23 Environ la moitié sont des césariennes programmées, l'autre des césariennes décidées en cours de travail.

REMI 2012 (28) 2 pp. 81-100 
dans des pays hors Afrique subsaharienne. En revanche, on n'observe aucune différence significative entre les taux de césarienne des femmes "nées en France » catégorisées comme «Africaines » et ceux des autres femmes nées en France.

Par ailleurs, si les variables sociodémographiques disponibles telles l'âge ou la parité (nombre d'enfants mis au monde) n'introduisent pas de variations significatives entre les groupes de femmes, il n'en va pas de même pour les problèmes de santé qu'ils soient connus antérieurement, gestationnels (apparaissant pendant la grossesse), ou survenant en cours de travail.

\section{Incidence des problèmes de santé sur les taux de césariennes}

L'étude de l'état de santé des femmes montre que les «Africaines immigrées » sont surreprésentées parmi les femmes ayant des grossesses pathologiques, à hauteur de $44 \%$ contre $25 \%$ en moyenne pour les autres femmes. Certaines particularités médicales sont relevées dans leurs dossiers. Parmi elles, $8,75 \%$ étaient porteuses du VIH-Sida (versus 0,7 à $1,9 \%$ pour les autres groupes), toutes ayant accouché d'un enfant vivant, dont aucun n'a été infecté cette année-là. Elles étaient également, et de façon significative, plus souvent atteintes du diabète (gestationnel ou préexistant), d'obésité sévère et morbide, d'hypertension artérielle, d'hémoglobinopathies et d'hépatites virales B et C.

Les pathologies maternelles qui se déclarent pendant le travail $(\mathrm{N}=485)$ ne sont pas liées aux pathologies de la grossesse décrites ci-dessus, mais concernent plutôt les situations maternelles préalables au travail ou apparaissant au cours de celui-ci et qui le compliquent. Parmi ces pathologies, on trouve le fait d'avoir été césarisée précédemment (femmes porteuses d'un utérus cicatriciel), le fait que le bassin maternel ne permette pas l'engagement du foetus, l'apparition d'une fièvre en cours de travail ou des anomalies de dilatation du col. Elles mènent à une césarienne dans $66 \%$ des cas, versus $9,5 \%$ si elles ne sont pas relevées ${ }^{24}$.

Les femmes «Africaines immigrées » sont celles qui présentent le plus fréquemment ces pathologies maternelles du travail (cette différence n'est pas retrouvée pour les femmes «Africaines nées en France ») notamment parce qu'elles ont été significativement plus souvent césarisées lors d'accouchements antérieurs à $2006(16,8 \%$ versus $11 \%$ de la population à l'étude) ${ }^{25}$. Or les femmes qui ont accouché par césarienne une première fois accouchent à nouveau par césarienne dans $64,3 \%$ des cas, alors que seuls $8 \%$ de celles ayant accouché normalement auparavant risquent d'être césarisées ${ }^{26}$.

Enfin, l'examen de la taille du bassin par radiologie, disponible dans 162 dossiers seulement ${ }^{27}$, montre sur cet effectif que près de neuf femmes sur dix «Africaines immigrées » présentent un bassin de taille inférieure à la « normale $»^{28}$ quand elles sont

$24 \mathrm{Khi}^{2}=689, \mathrm{p}=0,000$.

$25 \mathrm{p}=0,000$.

$26 \mathrm{Khi}^{2}=431,133, \mathrm{dl}=3 ; \mathrm{p}=0,000$.

27 Cet examen est rarement prescrit.

28 Le score de Magnin qui correspond à l'addition de deux des mensurations du bassin fixe une valeur en dessous de laquelle une césarienne est préconisée. Si ceci est parfois débattu, ce score continue néanmoins à faire référence (Lansac et al., 2003). 
un peu plus de six sur dix dans les autres groupes. Parmi les femmes catégorisées comme « Africaines », quoique nées en France, une seule présentait un bassin de taille inférieure à cette norme.

Au total, si les trajectoires de soins en maternité ne sont pas prévisibles d'un bout à l'autre, et que le déroulement d'une grossesse ne suffit pas à prédire l'accouchement, le moins bon état de santé constaté pendant la grossesse pour les femmes "Africaines immigrées » semble bien exercer une forte influence sur leur taux de césariennes à l'accouchement. De fait, pour l'ensemble de la cohorte, les femmes ayant eu des grossesses normales sur les plans maternel et fotal ont, dans 84,1\% des cas, accouché par voie basse, alors que, celles qui présentaient des pathologies, entraînant de surcroit des répercussions sur le développement fœtal, ne sont que $55 \%$ (45\% ont accouché par césarienne).

Néanmoins, la prise en compte des différentes variables dont nous disposions pondère ce premier constat. Des investigations plus poussées par des calculs de régressions logistiques permettent l'introduction des variables une à une pour en tester les effets (cf. Tableau 1). Ils confirment ces premières analyses, mais montrent qu'à terme persiste un effet ultime de la racisation de ces femmes sur leur probabilité d'être accouchées par césarienne.

Tableau 1 : Facteurs associés au risque d'accoucher par césarienne : l'importance des pathologies maternelles

\begin{tabular}{|l|l|l|l|}
\hline \multicolumn{1}{|c|}{ Variables explicatives } & \multicolumn{1}{|c|}{$\begin{array}{l}\text { Modalités (les valeurs de } \\
\text { référence sont en gras) }\end{array}$} & \multicolumn{1}{c|}{ Odd ratio } & \multicolumn{1}{c|}{$\mathrm{p}$} \\
\hline État de santé pendant la grossesse & $\begin{array}{l}\text { Normal } \\
\text { Pathologique }\end{array}$ & $\begin{array}{l}1 \\
2,433^{*}\end{array}$ & 0,000 \\
\hline « Racisation» & $\begin{array}{l}\text { « Non Africaine » } \\
\text { «Africaine » }\end{array}$ & $\begin{array}{l}1 \\
1,475\end{array}$ & 0,009 \\
\hline Pathologies Fœtales du travail & $\begin{array}{l}\text { Non } \\
\text { Oui }\end{array}$ & $\begin{array}{l}1 \\
1,334\end{array}$ & 0,031 \\
\hline Parité & $\begin{array}{l}\text { Primipare } \\
\text { Multipare }\end{array}$ & $\begin{array}{l}1 \\
0,472\end{array}$ & 0,000 \\
\hline Âge & $\begin{array}{l}\text { Moins de 38 ans } \\
\text { Plus de 38 ans }\end{array}$ & $\begin{array}{l}1 \\
1,790\end{array}$ & 0,002 \\
\hline Pathologies Maternelles du travail & $\begin{array}{l}\text { Non } \\
\text { Oui }\end{array}$ & $\begin{array}{l}1 \\
19,875\end{array}$ & 0,000 \\
\hline
\end{tabular}

Légende : L'odd ratio donne une valeur de prédiction statistique : l'OR inférieur à 1 montre que la variable testée n'influe pas sur la dépendante, s'il est proche de 1 l'influence existe, mais elle est faible alors qu'un OR plus grand que 1 indique que la modalité de la variable indépendante a autant de chances de se voir affecter la condition de la dépendante.

p correspond à la significativité des résultats : plus p est proche de zéro et plus le résultat est significatif (la limite de $\mathrm{p}=0,05$ a été fixée pour cette étude).

(*) Les femmes présentant une grossesse pathologique ont 2,43 fois plus de risque d'accoucher par césarienne que celles ayant eu une grossesse normale (population de référence, valeur 1), et ce à appartenance à une catégorie « racisante », parité, âge, et travail pathologique sur les plans maternel et fœtal, égaux. 
L'introduction des variables une à une dans le modèle de régressions bivariées, n'a fait que très peu varier les odds ratio, pas plus d'ailleurs que le test d'ordre d'introduction des variables, ce qui nous amène à ne présenter qu'un tableau ${ }^{29}$. Sa lecture ${ }^{30}$ montre que l'odd ratio le plus élevé concerne les pathologies maternelles du travail $(\mathrm{OR}=19,87)$. Elles entraînent donc vingt fois plus de risque d'être césarisées si elles sont relevées. Cependant, elles n'infirment pas le poids des autres variables testées qui ont, elles aussi, leur influence, même si elle est bien moindre. On note que la parité influe peu sur le modèle et de manière négative, car les multipares ont moins de risque d'accoucher par césarienne que les primipares (sauf pour celles ayant été césarisées précédemment). Les régressions logistiques binaires et multinomiales montrent qu'un effet des catégories racisantes persiste sur la probabilité qu'une femme soit césarisée même si l'odd ratio est proche de $1(\mathrm{OR}=1,48)$. Néanmoins, à état de santé égal à toutes les étapes de la trajectoire de soins, à âge égal, et à parité égale, les femmes catégorisées comme « Africaines » ont une probabilité supérieure et significative d'accoucher par césarienne que toutes les autres femmes.

Nos résultats statistiques attirent donc l'attention sur deux points essentiels : d'une part ils nous apprennent que le groupe des femmes catégorisées comme «Africaines» recouvre en fait deux sous-population bien distinctes, celles qui ont immigré et celles qui sont nées en France. Les premières ont un taux significativement plus élevé de césariennes que celles qui sont nées en France (qu'elles soient catégorisées comme "Africaines » ou d'une autre origine, y compris les Françaises d'ascendance française). D'autre part, il s'avère que cet écart tient principalement au fait que les femmes nées en Afrique Subsaharienne présentent un moins bon état de santé que l'ensemble de la population étudiée, qu'elles sont plus nombreuses à avoir un utérus cicatriciel (ce qui indique des césariennes antérieures) et enfin, que leurs tailles de bassins sont au-dessous des normes établies, ces mesures ne se retrouvant pas chez les femmes nées en France, mais catégorisées comme «Africaines ».

\section{USAGE ET EFFETS DES CATÉGORIES RACISANTES}

La catégorie «Africaines » est d'usage courant dans le langage des soignants des services de maternité franciliens, aussi bien lors de la présentation de dossiers médicaux que lors de discussions informelles à propos de leurs patientes ${ }^{31}$. Notre étude qualitative permet de déterminer que cette dénomination englobe tant les migrantes nées dans un pays d'Afrique subsaharienne, que leurs filles voire leurs petites-filles, quels que soient leur nationalité et leur lieu de naissance. Dans le même sens, les soignants évoquent des

29 Il s'est avéré que les tests de régression multinomiale, qui utilisent les mêmes variables que les tests de régression binaires, mais permettent de décliner les profils, valident les régressions binaires sans avoir plus de force explicative (ce qui est logique puisque les variables sont fortement inter corrélées).

30 Ce modèle est explicatif dans $84 \%$ des cas, avec un $\mathrm{Khi}^{2}$ toujours significatif pour $\mathrm{p}=0,000$, mais avec 686 occurrences sur 1560 expliquées (log de vraisemblance), ce qui se traduit aussi par un taux de Nagelkerke de $42,4 \%$.

31 Sur les cinquante-six soignants interrogés, un seul a remis en cause cette catégorisation. 
«Africains » quand ils parlent des conjoints des femmes qu'ils décrivent, semblant exclure du groupe «Africain » celles qui sont en couple « mixte ».

Plusieurs registres sont convoqués pour construire les frontières de la catégorie : leurs cultures et leurs modes de vie, les réseaux sociaux auxquels elles appartiennent, leurs positionnements par rapport aux soins, mais également des éléments d'ordre biologique $^{32}$. Au fil des entretiens, nous avons repéré que les niveaux de revenus et d'éducation supposés peuvent intervenir pour décliner des sous-catégories. Ainsi, les " Maliennes » désignent des femmes supposées d'origine rurale et relevant de structures familiales traditionnelles. Elles sont opposées aux « Camerounaises », prétendument citadines et au niveau d'éducation plus élevé, et distinguées des « Congolaises » arrivées plus récemment et plus isolées ${ }^{33}$. Le revenu sert de base de classement pour distinguer les «Africaines », décrites en situation de précarité ou à l'inverse appartenant aux classes sociales les plus aisées, des «Antillaises », catégorie qui regroupe toutes les femmes que les soignants voient comme « noires » et situent dans une classe moyenne, même si elles sont nées dans un pays d'Afrique.

Il demeure que ces sous-catégories ne présentent ni la même consistance ni la même stabilité, dans le discours des soignants, que celles d' " Africaines », désignation qui les autorise à parler fréquemment, de « diabètes africains », de « petits Africains qui meurent plus à la naissance » ou de « femmes noires dont les fins de grossesse sont plus à risque ». Ces discours, souvent inspirés par la généralisation d'études épidémiologiques américaines, sont de plus en plus courants dans le langage quotidien.

À propos d'observations et d'entretiens menés dans trois services franciliens, Nacu s'interroge sur la fonction du culturalisme dans les relations de soins en maternité (Nacu, 2011). Si nos résultats recoupent les siens à bien des égards, pour ce qui concerne les soins (cure) obstétricaux, c'est bien une " racisation » des patientes qui est opérée. Elle participe d'une banalisation des catégories « raciales » réifiées, telles qu'on peut les retrouver dans le recensement de groupes « à risque » (Bonnet, 2009 ; Fujimara 2009).

\section{La « race » au service du discours sur la « mécanique obstétricale »}

Dans le mémoire de fin d'études d'une sage-femme, rédigé en 2011 et fort précis sur d'autres points, se trouve la description suivante : " Le bassin osseux des Africaines est réputé comme étant transversalement rétréci. Il s'avère que le détroit supérieur est de type anthropoïde. Le diamètre transverse utile se trouve à la limite inférieure par rapport aux normes des bassins des Caucasiennes » (Bras, 2011 : 19). Cette description correspond à celle proposée par Caldwell et Moloy en $1933^{34}$ et qui est toujours enseignée

32 D'autres catégories de femmes sont construites sur le même principe et avec les mêmes imprécisions : les « Asiatiques », les « Chinoises », les « Maghrébines », les « Mexicaines », etc.

33 Ce type de distinction avait été relevé par Poiret (1996).

34 Les femmes d'Afrique subsaharienne auraient majoritairement des bassins ovales ou " anthropoïdes », avec pour certaines d'entre elles des bassins " androïdes », triangulaires et plus semblables à ceux des hommes, donc ne permettant pas un accouchement voie basse (CaldwellMoloy, 1933). Ces notions sont retrouvées dans les entretiens, avec quelques confusions entre ces deux termes dont la prononciation est assez proche. 
dans les cours d'obstétrique. Retrouver ainsi décrites les supposées caractéristiques des femmes racisées comme «Africaines », en les opposant sans discussion ni précision aux «Caucasiennes », traduit clairement les représentations que les formations médicales inculquent aux soignants et qu'ils diffusent à leur tour.

Nos analyses prennent d'autant plus de sens que la littérature obstétricale nous apprend que, lorsque les équipes soignantes prennent du recul par rapport à leurs pratiques et s'adaptent à des situations nouvelles ou différentes, les taux de césarienne reculent sans que ne s'accroissent pour autant la mortalité maternelle ou/et la mortalité périnatale des nourrissons. La première équipe hospitalière parisienne, après avoir constaté un taux de césariennes deux fois supérieur chez les femmes originaires du Mali et du Sénégal, avait préconisé une attente plus longue avant de décider d'une éventuelle césarienne en cours de travail. Les médecins considéraient que si les bassins étaient plus petits, la tête du bébé était aussi plus petite, plus malléable, que sa position dans le bassin était différente, et les nouveau-nés de poids inférieurs. Cet ensemble de caractéristiques entraînant certaines spécificités du travail de l'accouchement, il convenait de les respecter et de ne pas prendre de décisions trop hâtives. Les résultats ont été immédiats : l'année suivante, le taux de césariennes pour les parturientes "Africaines » a été abaissé à $13 \%$. Les césariennes en cours de travail ont diminué de moitié, et les césariennes programmées ont été divisées par quatre (Pigné et al., 1984 et 1985). Une étude menée au Havre, entre 1976 et 1986, a montré que le taux de césarienne pour la population " africaine » avait aussi diminué, de $9,3 \%$ à $7,5 \%$ (en dépit d'une augmentation de 4,5 à $9,5 \%$ pour la population générale) en suivant les recommandations de l'équipe parisienne (Col, 1989). De leur côté, Pambou et ses collaborateurs enregistrent une baisse spectaculaire des taux dans la région sud de Paris entre 1981 et 1984 (18,8\% versus 5,6\%), sans augmentation de la morbidité maternelle ou néonatale à partir du moment où, disent-ils « les équipes obstétricales font l'effort de s'adapter » et renoncent aux " césariennes systématiques pour bassin rétréci ou utérus cicatriciel » (Pambou et al., $1996: 371)$.

Si l'équipe de Pigné (1984) n'interroge pas les différences de tailles de bassins, les tenant sans doute pour « naturelles », Col (1989), pour sa part, tente d'en comprendre les causes et il avance plusieurs possibilités : la nutrition, les variations hormonales, des facteurs dits environnementaux (incluant l'obligation de porter des charges lourdes) et les facteurs génétiques. En revanche, à la fin des années 1990, dans l'étude toulousaine qui cherchait à identifier les risques périnataux liés à « l'origine ethnique des mères » (GayralTaminh et al., 1999), les chercheurs ont aussi trouvé un taux de césarienne plus élevé pour les femmes «d'origine africaine » (qui sont dans leur étude des femmes immigrées d'Afrique subsaharienne), mais sans retrouver de poids plus petits des nouveau-nés. Ils ont référencé les études précédentes et ont souligné « l'importance de prendre en compte l'origine ethnique, tant pour la prise en charge obstétricale des patientes que pour les études épidémiologiques », sans proposer d'adopter une conduite plus attentiste (GayralTaminh et al., 1999 : 470).

Notre étude quantitative montre, comme toutes les études parues depuis sa réalisation, que les taux de césariennes des femmes catégorisées comme «Africaines » restent supérieurs à ceux des autres femmes. Mais nous montrons aussi que ce sont des variables imputables d'abord aux états de santé des femmes nées dans un pays d'Afrique 
Subsaharienne, et ensuite au déroulement de leur travail, qui sont les plus explicatives. En effet, les femmes «Africaines immigrées » présentent, dans notre étude, des états de santé défavorables. Les données disponibles ne nous ont pas permis d'étudier si les femmes étaient arrivées en France porteuses de ces pathologies ou si elles les ont développées après leur arrivée. La dégradation de leur état de santé du fait de conditions socio-économiques, juridiques et affectives défavorables en migration n'est pas à écarter et l'étude de ces processus serait fort instructive. Par ailleurs se posent pour elles les problèmes de taille de bassin plus petite et de défaut de progression du fœtus dans le bassin. Les pratiques médicales sont aussi interrogées au travers de leur taux élevé d'utérus cicatriciels, conséquence d'une, voire plusieurs césariennes antérieures, dont les causes ne sont pas renseignées. Cependant, il faut souligner que le moins bon état de santé des femmes «Africaines immigrées » n'est pas corrélé à un moins bon état de santé de leurs fœtus/nouveau-nés dans notre étude, qui ne présentent pas non plus de poids différents des autres. Ce dernier élément, s'il est positif dans le sens où une corrélation entre faible poids de naissance et risque de mortalité périnatale a été montrée (Williams et al., 1989), pourrait néanmoins expliquer un engagement des fœus dans les bassins, moins aisé. Enfin, nous ne pouvons pas exclure que l'introduction de données socio-économiques, si elles avaient été disponibles, aurait pu faire varier certains de nos résultats.

Notre attention a été attirée à de multiples reprises par les amalgames faits entre plusieurs notions par les soignants, qui participent, à notre sens, des processus de racisation et influencent les décisions cliniques. Pour ce qui réfère à la « mécanique obstétricale », l'absence de directives claires de la part des chefs de service a pour conséquence de laisser plus de place aux représentations dans les orientations et décisions cliniques, avec des pratiques très variables d'un clinicien à un autre. À propos des femmes qu'ils catégorisent comme " Africaines », les cliniciens exerçant en salle d'accouchement parlent de "petits bassins » et qualifient cette spécificité de " génétique » (ou "d'ethnique »). De façon liée à ces représentations, certains décident d'orienter systématiquement vers une césarienne quand d'autres proposent d'attendre plus longtemps pour l'engagement du fœtus, favorisant un accouchement par voie basse même en cas d'utérus cicatriciel et de bassin estimé petit. Ce qui ne varie pas en revanche, c'est que des différences entre «Africaines » ne sont qu'exceptionnellement évoquées et que les facteurs qui ont pu influer sur leurs tailles de bassin (dénutrition, pubertés et maternités précoces, épidémies de poliomyélites, etc.) ne sont que trop peu souvent discutés et, a fortiori, recherchés. Or, notre étude en scindant le groupe des «Africaines » en deux - femmes immigrées versus femmes nées en France - montre que seules les premières sont concernées par ces taux de césarienne élevés et par ces différences de "mécanique obstétricale ». Nous pensons donc que les ceintures pelviennes des femmes nées en France de parents «Africains » ne présentent pas les caractéristiques de celles de leurs mères, ce que confirment les quelques radios du bassin que nous avons retrouvées (qui constituent cependant un échantillon trop petit pour être probant). Ceci corrobore les études menées aux États-Unis, qui ont montré des différences de croissance entre les enfants de Japonais immigrés aux États-Unis et les enfants japonais au Japon. Les chercheurs ont constaté une variation de leur périmètre céphalique en une génération (Kano et Chung, 1975). Une étude française a trouvé des résultats similaires pour les enfants d'origine chinoise (Roville-Sausse, 1991). Dans le cas des femmes catégorisées comme «Africaines », l'impact que peuvent avoir sur leur santé les facteurs alimentaires, les facteurs environnementaux et leurs conditions de vie 
en général est trop rarement interrogé. Domine plutôt une interprétation fondée sur les représentations stéréotypées de prétendues caractéristiques génétiques, anatomiques ou/ et physiologiques.

\section{Racisation et application du « protocole de terme ethnique » : des effets discriminatoires?}

À trois exceptions près, les soignants interrogés appartenaient à des équipes ayant mis en place un protocole de «terme ethnique », ce qui confirme que la pratique en était fort répandue. A priori, les recommandations émises en décembre 2011 par le Collège national des gynécologues et obstétriciens français devraient la faire régresser, voire cesser (CNGOF, 2011). Le type de femmes orientées vers ce protocole varie selon les équipes : soit il ne s'agit que des femmes catégorisées comme "Africaines », soit y sont incluses toutes les femmes racisées comme " Noires » que leurs origines soient supposées être des Antilles, d'Haïti, du Sri Lanka, ou encore de l'archipel des Comores, mais pas les Malgaches ${ }^{35}$. Selon l'interprétation qu'un médecin ou une sage-femme se fait de l'origine ou de la couleur de leur peau, des femmes sont donc suivies plus précocement d'une semaine que les autres en fin de grossesse (quarante semaines d'aménorrhées au lieu de quarante et une) et, en conséquence, leur accouchement est au besoin déclenché à moins de quarante et une semaines (on compte un maximum de cinq jours après le terme considéré).

Les discours des soignants à propos de ce protocole sont imprécis. Peu d'entre eux ont lu l'étude de Papiernick (Papiernick et al., 1990) et le bien-fondé du protocole leur semble évident. Ils arguent d'une « physiopathologie » différente qui concernerait soit la durée de gestation foetale, soit la maturité placentaire. Pour eux, appliquer ce protocole permettrait de baisser la mortalité foetale périnatale. Nous avons poursuivi ces hypothèses en demandant à nos interlocuteurs pourquoi les enfants " métis » n'étaient pas surveillés de la même façon. Cette question a entraîné une grande confusion, car la plupart des cliniciens n'avaient pas envisagé cette option. Certains ont évoqué un terme «demi-ethnique », d'autres ont avancé que ceci se faisait peut-être « aux Antilles ». Mais globalement, la noblesse de la cause l'emportait sur la réflexion. L'impact d'autres facteurs, comme la qualité de vie des femmes et/ou leurs conditions socio-économiques, n'était pas interrogé.

Par ailleurs, les soignants informent rarement leurs patientes de ce traitement différentiel, ou alors ils présentent les choses sur le ton de l'humour : «Je leur dis, on prend soin de vous, on vous voit plus souvent que les autres. Vous ne pourrez vous en plaindre! » (Sage-femme). Et de préciser qu'ainsi les femmes «le prennent bien ». Cette absence d'explicitation se poursuit jusqu'au moment de programmer le déclenchement, les femmes n'étant qu'exceptionnellement informées des raisons sous-jacentes à cette décision clinique. Cependant, nous avons trouvé certaines variations dans les pratiques soignantes concernant le protocole de «terme ethnique». Notre étude quantitative montre qu'un petit nombre de femmes, pourtant incluses dans ce protocole, ont pu poursuivre leur grossesse jusqu'à quarante et une semaines voire jusqu'à quarante-deux semaines. Nous avons également constaté que les fotus des femmes déclenchées présentaient plus

35 Les soignants interrogés les considèrent comme « Asiatiques ». 
de pathologies de fin de la grossesse ou du travail, ce qui peut justifier la décision du déclenchement.

Il n'en reste pas moins que le protocole de «terme ethnique » traduit le franchissement d'un pas dans le processus de racisation des femmes puisqu'il concerne une population spécifique qui n'est désignée ni par l'ensemble des pays dont elle serait originaire ni par une maladie, mais par une couleur de peau « Noire ». De plus, il ne concerne pas les femmes catégorisées comme « non noires » même si leur conjoint est « Noir », ce qui rejoindrait, si l'on en fait une lecture foucaldienne, une volonté de gouverner plus spécifiquement le corps de certaines. Ceci va dans le sens du ressenti des femmes qui se disent victimes d'un trop grand nombre de césariennes et affirment que les médecins français souhaiteraient, par ce biais, contrôler leur fertilité. Une perception que les soignants récusent immédiatement en la qualifiant de paranoïaque.

Au total, la population ciblée n'est pas bien définie, elle est laissée à la libre interprétation du soignant. Les femmes ne sont pas informées de ce protocole qui les concerne, mais qui a été instauré sans consultation préalable d'instances sanitaires ni de comité d'éthique. Il n'a pas été évalué a posteriori par des études, sauf Gayral Taminh et ses collaborateurs qui notent en 1999 qu'ils ne retrouvent pas la différence de temps de gestation dans la population qu'ils ont étudiée à Toulouse (Gayral-Taminh et al., 1999), et un mémoire comparant "l'état néonatal des enfants Africains et Caucasiens ", dans lequel l'auteur confirme à partir de ses observations « la durée moyenne de grossesse des africaines d'une semaine plus précoce que les caucasiennes » (Sackett, 2001 : 46) ${ }^{36}$. Enfin, en 2011, le CNGOF précise que : « Chez les femmes noires africaines, la mortalité fœtale n'est pas significativement augmentée en fin de grossesse. Il n'existe pas de données pour modifier les pratiques en cas de grossesse prolongée dans cette population » (CNGOF, 2011 : 703). Cette dernière phrase ne laisse même pas deviner que le protocole puisse exister depuis dix ans. La question se pose donc de savoir si ces traitements différentiels peuvent être analysés comme des pratiques discriminatoires, c'est-à-dire des différences de traitement illégitimes et produisant des effets négatifs pour ceux qui les subissent.

Dans le domaine des soins, il est très difficile et fort mal reçu d'évoquer la discrimination à l'égard des patients. Nous avons pu le vivre personnellement et l'expérimenter lors de discussions professionnelles. Cette conviction d'être hors du racisme et des discriminations, s'appuie sur une certitude, partagée par la quasi-totalité des personnels médicaux, d'agir au mieux pour le bien des personnes soignées (principe de bienfaisance) (Cognet et al., 2009). Même si les traitements réservés aux femmes racisées ne consistent, en l'état actuel de nos résultats, qu'en quelques consultations prénatales supplémentaires, en un éventuel déclenchement plus précoce et en un taux supérieur de césarienne qui, quoique net dans nos analyses statistiques (cf. Tableau 1), reste relativement expliqué par des états de santé différents, il est primordial de poursuivre ces investigations et d'examiner avec la plus grande attention les conséquences de ces pratiques.

36 Ceci ne veut pas dire qu'il n'y ait pas eu d'autres mémoires sur le sujet. Nous citons cet exemple qui nous permet de réintroduire les critiques formulées plus haut sur les imprécisions ayant cours. 


\section{CONCLUSION}

L'identification de différences physiques ou physiologiques supposées de certaines femmes, combinée à l'imputation de différences culturelles, à des perceptions plus ou moins stéréotypées de leurs modes de vie et à l'identification de " problèmes " posés par certaines patientes aux professionnels, aboutissent à la construction de catégories ethnicisantes et racisantes en périnatalité. La catégorie " Africaines » est rarement scindée en sous-catégories par les soignants, mais, en fonction des professions et des postes occupés, le contenu qui lui est donné diffère. En nous intéressant aux discours des médecins et des sages-femmes à propos de leurs pratiques ou observations cliniques, donc aux discours les plus médicaux, ainsi qu'aux protocoles cliniques spécifiques, nous constatons que ces catégories confèrent aux femmes une altérité essentialisée qui semble transmissible de génération en génération. Ces processus de racisation, ayant des effets sur les trajectoires de soins des femmes, se développent dans un domaine d'activité qui se pense hors du racisme et veut combattre l'exclusion en travaillant au bon état de santé global des individus. Les soignants affirment d'ailleurs respecter, voire favoriser, le principe d'égalité pour tous dans l'accès aux soins. Les catégories qu'ils mobilisent n'en traduisent pas moins une habitude médicale de penser des différences entre des groupes humains, différences qu'ils qualifient volontiers d'《 ethniques » ou de " génétiques ». Ces discours, qui se basent sur leurs pratiques professionnelles, sont acquis au cours des enseignements reçus sur les bancs de la faculté ou sur les terrains de stage hospitaliers. Pourtant, face à eux, des femmes qui s'identifient comme «Africaines » ou des associations qui les représentent, remettent en cause de façon récurrente les pratiques médicales, en particulier les césariennes, qu'elles perçoivent comme un soin discriminatoire à leur égard.

Des traitements différentiels sont proposés aux patientes " Africaines », issus de pratiques individuelles au sein de la relation de soins, mais aussi de protocoles spécifiques. Notre étude permet de pointer un certain nombre de différences dans l'état de santé des femmes «Africaines » ayant migré. Elles sont plus souvent porteuses de pathologies que toutes les autres femmes étudiées, et ont des taux de césariennes très supérieurs. Ces derniers semblent tout particulièrement liés à des éléments affectant le bon déroulement du travail (taille de bassin, césariennes antérieures) qui ne sont pas corrélés au mauvais état de santé. Ces données seraient à étudier de plus près, car elles semblent recouper les situations décrites dans les années 1980, même si, nous l'avons démontré, les situations des unes et des autres ne sont plus tout à fait comparables et que leurs nouveaunés présentent aujourd'hui des poids similaires à ceux des autres groupes étudiés. En revanche, les femmes catégorisées comme «Africaines nées en France » ne présentent pas ces différences, ni d'état de santé ni de mode d'accouchement. Mais cette distinction n'est pas toujours claire dans l'esprit de soignants.

L'état de santé moins bon des femmes « Africaines immigrées » peut être questionné sous l'angle des disparités d'états sanitaires de par le monde et des variations de conditions économiques des États, mais ce ne sont pas les seules explications. En effet, les difficultés de communication, les prises en charge tardives des femmes et leur moins bonne connaissance du système de soins sont identifiées comme des facteurs de rique par les épidémiologistes étudiant la mortalité maternelle en France (Bouvier Colle et al., 2008), qui préconisent la correction de ces inégalités. Il nous semble également indispen- 
sable de poursuivre l'étude des processus de racisation, et de leurs conséquences en termes de risques de discrimination des patientes, à chaque étape des soins et de l'accès aux soins.

En effet, si nos données quantitatives ne permettent pas de montrer de manière certaine une discrimination particulière via des taux élevés de césariennes, puisque ceux-ci sont largement expliqués par des états de santé différents, elles n'en interrogent pas moins l'état des connaissances sur des " mécaniques obstétricales 》 spécifiques et sur leurs processus, insuffisamment étudiés. Notre étude qualitative, quant à elle, montre précisément que les soignants, qui ont l'habitude de penser le corps comme un corps biologique, appliquent sans les interroger des protocoles médicaux qui s'appuient sur des déterminants pouvant entraîner de la discrimination en raison d'origines supposées. Ainsi, un praticien peut déclencher plus tôt l'accouchement d'une femme à cause de la couleur de sa peau, parce que de tels protocoles ont été produits par sa hiérarchie hospitalière. Or il ne semble ni pertinent d'englober des femmes nées en France et des femmes immigrées dans des protocoles médicaux spécifiques, sous prétexte d'origines « africaines » communes, ni juste d'amalgamer des femmes au nom d'une couleur de peau perçue, en présumant génétiques et héréditaires certaines caractéristiques qui relèvent bien plus de l'état de santé et des conditions de vie. De plus, l'ensemble des données que nous avons présenté montre que les justifications cliniques soutenant ces pratiques et protocoles différentiels, voire discriminants, se sont trouvées, au fil de l'histoire récente de l'obstétrique, totalement invalidées quelques années après leur publication. Ces amalgames en revanche, contribuent bien à fabriquer de la « race ». À terme, la déconstruction de l'imputation d'altérité pourrait permettre d'éviter les traitements différentiels basés sur ce type de variables, surtout lorsqu'ils sont discriminatoires, pour se consacrer à de véritables corrections des inégalités de santé. 


\section{Références bibliographiques}

ABOUKRA Audrey et NOIVILLE Christine (2011) Les médicaments « raciaux » ou « ethniques » : manoeuvre commerciale ou enjeu de santé publique, in Guillaume Canselier et Sonia DesmoulinCanselier Éds., Les catégories ethnoraciales à l'ère des biotechnologies. Droit, sciences et médecin face à la diversité humaine, Vol. 24, Paris, Société de législation comparée, pp. 55-76.

ALEXANDER Greg R., BARUFFI Gigliola, MOR Joanne M., KIEFFER Edith C. and HULSEY Thomas C. (1993) Multiethnic Variations in the Pregnancy Outcomes of Military Dependants, American Journal of Public Health, 83 (12), pp. 1721-1725.

BA Marième (2000) La dépression du ventre. Réactions dépressives après l'accouchement chez les femmes immigrées originaires d'Afrique de l'Ouest, in Tobie Nathan, L'enfant Ancêtre, Paris, Éditions La pensée sauvage, 300 p.

BARRÂT Jacques (1986) Les césariennes chez les Africaines, La lettre du Gynécologue, 51, pp. 1-5.

BENOIT Amélie (2004) Maternité chez la femme d'Afrique Noire au CHU de Rouen, Mémoire pour le D.E. de sage-femme, CHU de Rouen, non publié.

BLONDEL Béatrice et KERMARREC Morgane (2010) Les naissances en 2010 et leur évolution en 2003, DRESS, Études et résultats, 775, pp. 1-8.

BONNET Doris (2009) Repenser l'hérédité, Paris, Éditions des archives contemporaines, 116 p.

BOUVIER-COLLE Marie-Hélène, DENEUX-THARAUX Catherine and PHILIBERT Marianne (2008) Can excess maternal mortality among women of foreign nationality be explained by suboptimal obstetric care?, British Journal of obstetrics and gynecology, 115 (11), pp. 14111418.

BRAS Camille (2011) Regards sur la césarienne. Témoignages de femmes originaires d'Afrique Subsaharienne. Mémoire pour l'obtention du diplôme d'État de sage-femme, Université de Rouen, non publié, 92 p., [en ligne]. URL : http://www. dumas.ccsd.cnrs.fr/docs/00/65/14/82/ PDF/BRAS_Camille.pdf

CARDE Estelle (2011) La race, de la catégorie sociale à l'objet d'étude épidémiologique, in Guillaume Canselier et Sonia Desmoulin-Canselier Éds., Les catégories ethnoraciales à l'ère des biotechnologies. Droit, sciences et médecin face à la diversité humaine, Vol. 24, Paris, Société de législation comparée, pp. 78-88.

CATHEBRAS Pascal et PERROT Jean-Luc (2001) Qu'est-ce qu'un caucasien ? Race et ethnicité dans la littérature médicale, La presse médicale, 30 (20), pp. 1012-1013.

CNGOF (2011) Recommandations pour la pratique clinique, Grossesse prolongée et terme dépassé, Journal de gynécologie, obstétrique et biologie de la reproduction, 40 (8), pp. 693-962.

COGNET Marguerite, ADAM-VÉZINA Émilie and BASCOUGNANO Sandra (2009) Discrimination in Health Care Services, Paper presented at the 8th Global Conference Making Sense: Health, Illness and Disease, [en ligne]. URL: http://www.inter-disciplinary.net/ wp-content/uploads/2009/06/Adam-Vezina-Paper.pdf

COGNET Marguerite et BASCOUGNANO Sandra (à paraître) Soins et altérité : analyse comparée des regards et des pratiques des soignants, Alterstice.

COL Jean-Yves (1989) L'accouchement en France des patientes nées en Afrique Noire, Revue française de gynécologie obstétrique, 84, pp. 425-434.

DELFRAISSY Jean-François (1999) Rapport 1999 sous la direction du Professeur Delfraissy. Prise en charge thérapeutique des personnes infectées par le VIH, recommandation du groupe d'experts, Paris, Flammarion, $231 \mathrm{p}$.

DE RUDDER Véronique, POIRET Christian et VOURC'H François (2001) L'inégalité raciste : l'universalité républicaine à l'épreuve, Paris, Presses universitaires de France, 213 p.

DIN-DZIETHAM Rebecca and HERTZ-PICCIOTTO Irva (1998) Infant Mortality Differences between Whites and African-Americans: the Effect of Maternal Education, American Journal of Public Health, 88 (4), pp. 651-656. 
DOZON Jean-Pierre et FASSIN Didier (2001) Critique de la santé publique, une approche anthropologique, Paris, Balland, $362 \mathrm{p}$.

FASSIN Didier (2006) Du déni à la dénégation. Psychologie politique de la représentation des discriminations, in Didier Fassin et Éric Fassin Éds., De la question sociale à la question raciale?, Paris, La Découverte, pp. 133-157.

FUJIMARA Joan H. (2009) Circumventing or Reifying Race? The Use of Ancestry versus Race in Biomedical Genetic Research. Communication non publiée au Colloque international ANR «BIOSEX » Les catégories de sexe et race dans les sciences biomédicales contemporaines, 29 mai 2009, Université Paris-Sorbonne.

GAYRAL-TAMINH Martine, ARNAUD Catherine, PARANT Olivier, FOURNIER Alain, REME Jean-Michel, GRANDJEAN Hélène (1999) Grossesse et accouchement des femmes originaires du Maghreb et d'Afrique Noire suivies à la maternité du CHU de Toulouse, Journal de gynécologie obstétrique et biologie de la reproduction, 28 (5), pp. 462-471.

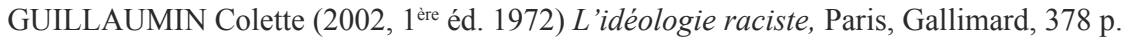

HENN Wolfram (2011) Ethnomédecine et justice dans l'ère post-génomique : entre soins de santé pesonnallisés et racisme high-tech, in Guillaume Canselier et Sonia Desmoulin-Canselier Éds., Les catégories ethnoraciales à l'ère des biotechnologies. Droit, sciences et médecin face à la diversité humaine, Vol. 24, Paris, Société de législation comparée, pp. 33-40.

KANO K., CHUNG C.-S. (1975) Do American born Japanese still grow faster than native Japanese?, American Journal of Physical Anthropology, 43 (2), pp. 187-194.

LANSAC Jacques, BERGER Christian et MAGNIN Guillaume (2003) Obstétrique pour le praticien, Paris, Édition Masson, 469 p.

MABE Brigitte (1985) Fécondité, grossesse, accouchement dans les sociétés négro-africaines, quelques points de repères anthropologiques, Migration santé, 44, pp. 7-15.

MORO Marie-Rose (2002) Enfants d'ici venus d'ailleurs, Paris, La découverte, 191 p.

NACU Alexandra (2011) À quoi sert le culturalisme ? Pratiques médicales et catégorisations des femmes « migrantes » dans trois maternités franciliennes, Sociologie du travail, 53, pp. 109-130

OLIVIER DE SARDAN Jean-Pierre (1995) La politique du terrain. Sur la production des données en anthropologie, Enquête, 1, pp. 71-112.

PAMBOU Olivier, KONGO P. et UZAN Serge (1996) L'accouchement des noires africaines à la maternité Guy Le Lorier de l'hôpital Tenon. Étude rétrospective de 781 accouchements de 1989 à 1991, Médecine d'Afrique Noire, 43 (6), pp. 371-377.

PAPIERNICK Emile, ALEXANDER G.-R. and PANETH Nigel (1990) Racial Differences in Pregnancy Duration and its Implications for Perinatal Care, Medical Hypothesis, 33, pp. 181-186.

PATEL Roshni, STEER Philip, DOYLE Pat, LITTLE Mark P. and ELIOTT Paul (2003) Does Gestation vary by ethnic group? A London-based study of over 122.000 pregnancies with spontaneous onset of labour, International Journal of Epidemiology, 33, pp. 107-113.

PIGNE Alain, KLEIN M., DIRATH G. et BARRAT J. (1985) L'accouchement de la femme d'Afrique Noire en France. Un aspect particulier : la césarienne, Migration santé, 44, pp. 31-34.

PIGNE Alain, KLEIN M., DIRATH G. et BARRAT J. (1984) L'accouchement de la femme d'Afrique Noire en France. Un aspect particulier : la césarienne, Journal de Gynécologie, Obstétrique et Biologie de la reproduction, 13 (7), pp. 812-816.

POIRET Christian (2011) Les processus d'ethnicisation et de raci(ali)sation dans la France contemporaine : Africains, Ultramarins et « Noirs », Revue Européenne des Migrations Internationales, 27 (1), pp. 107-127.

POIRET Christian (2010) Le retour de la catégorie " Noirs » dans l'espace public français, Migrations société, 22 (131), pp. 69-85.

POIRET Christian (1996) Familles africaines en France : ethnicisation, ségrégation et communalisation, Paris, l'Harmattan, $448 \mathrm{p}$. 
ROBERTS Dorothy (2011) Qu'est-ce qui ne va pas avec la « médecine raciale » ? Génétique, pharmacologique et égalité, in Guillaume Canselier et Sonia Desmoulin-Canselier Éds., Les catégories ethnoraciales à l'ère des biotechnologies. Droit, sciences et médecin face à la diversité humaine, Vol. 24, Paris, Société de législation comparée, pp. 41-53.

ROVILLE-SAUSSE Françoise (1991) Les rythmes de croissance dans une population d'enfants d'origine chinoise vivant à Paris, Bulletins et Mémoires de la Société d'anthropologie de Paris, Nouvelle Série, 3 (3-4), pp. 201-210.

SACKETT Myriam (2011) Comparaison et évaluation de l'état néonatal des enfants Africains et Caucasiens. Intérêt d'une prise en charge plus précoce, Université de médecine Paris Descartes, [en ligne]. URL : http://dumas.ccsd.cnrs.fr/docs/00/62/39/29/PDF/memoire_esfbaudelocque_ sackett.pdf

SAUVEGRAIN Priscille (2011) La migration influence-t-elle le rapport des parturientes à la maternité publique française ?, Migrations santé, 142-143, pp. 29-61.

SAUVEGRAIN Priscille (2010) Différence de traitement et traitements différentiels. Les trajectoires de soins des femmes "Africaines » en maternité publique, Thèse de doctorat ss la dir. de Catherine Quiminal, Véronique De Rudder et et sous la guidance de Marguerite Cognet, Université Paris Diderot, non publiée.

SCHOR Paul (2003) Statistiques de la population et politique des catégories aux États-Unis au XIXe siècle. Théories raciales et questions de population dans le recensement américain, Annales de démographie historique, 105 (1), pp. 5-22.

SIMON Patrick (1997) La statistique des origines : l'ethnicité et la « race » dans les recensements aux États-Unis, Canada et Grande-Bretagne, Sociétés contemporaines, 26 (2), pp. 11-44.

TRUDEL Robert et ANTONIUS Rachad (1991) Méthodes quantitatives appliquées aux sciences sociales, Montréal, CEC, $545 \mathrm{p}$.

WILLIAMS R.-L., CREASY R.-K., CUNNINGHAM G.-C., HAWES, W.-E., NORRIS, F.-D. and TASHIRO M. (1989) Fetal growth and perinatal viability in California, Obstetrics and Gynecology, 59, pp. 937-943. 


\title{
La santé maternelle des « Africaines » en Île-de-France : racisation des patientes et trajectoires de soins
}

\author{
Priscille Sauvegrain
}

Le personnel médical et paramédical des services de maternité franciliens parle souvent des «Africaines » comme d'une catégorie de femmes présentant des caractéristiques spécifiques. Nous présentons et discutons ici les résultats d'une recherche qui avait pour objectif d'analyser les processus et les effets de cette catégorisation. Nous montrerons que ces processus ne sont pas neutres sur les trajectoires de soins. En particulier, nos analyses quantitatives montrent des taux de césariennes élevés pour les femmes «Africaines » immigrées, même après correction d'autres variables testées comme l'état de santé. Tous nos résultats convergent pour montrer les risques inhérents à l'usage de catégories « racisantes » de patientes. Il permet en effet l'application de protocoles médicaux fondés sur des imprécisions et des amalgames, et qui ne corrigent pas les éventuelles inégalités de santé périnatale.

\section{Reproductive Health of “African”-perceived Women in Paris Area: Racial Categorisations and Medical Course}

\author{
Priscille Sauvegrain
}

Care providers, in public maternity hospitals in Paris area, often spoke about "African" women as a category with specific characteristics. This article presents and discusses a research analysing the processes of racial categorisations and their impact on the medical course. Our quantitative research pointed out a high rate of C-sections for the "African" women, when they're migrants, even after correcting other potential influences like state of health. We show that differential medical protocols proposed to reduce inequalities in health do not reach their goal, and sometimes induce discriminations. But we constantly show how sociological analyses help deconstructing medical stereotypes or ideologies that have been embodied by the medical staffs.

\section{La salud materna de las «africanas» en Île-de-France: racialización de las mujeres y trayectorias de cuidados}

Priscille Sauvegrain

Se suele oír al personal médico y paramédico de los servicios de maternidad de la región parisina hablar de «africanas» como una categoría de mujeres con características específicas. En este trabajo se presentan y se discuten los resultados de una investigación sobre los procesos y efectos de esta categorización. Vamos a mostrar que estos procesos no son neutrales en las trayectorias de cuidados. En particular, nuestros análisis cuantitativos muestran altas tasas de cesárea para las mujeres «africanas» inmigrantes, aun después de examinar las variables que hemos obtenido en los análisis. En conjunto, nuestros resultados convergen para mostrar los riesgos inherentes al uso de las categorías «racializantes» de los pacientes, tanto en términos de imprecisión y amalgamas que permiten la aplicación de los protocolos sin justificación como en término de no corrección de las desigualdades en la salud perinatal. 